\title{
Theme: Adolescent Pediatrics
}

\section{Tdap vaccination strategies for preventing pertussis in adolescents ( $\mathrm{J}$ Adolesc Health. 2018;62:661-6)}

In recent times, there has been a global resurgence of pertussis. High Tdap vaccine coverage in adolescents protects against pertussis and decreases the reservoir for transmission to vulnerable population. This retrospective cohort study among 165,541 adolescents (age 11-18 y) enrolled in heath care systems from 2005-12 was conducted in USA to determine the vaccine effectiveness (VE) of Tdap in those who had received only acellular vaccine, and in those who had received at least one dose of whole cell vaccine by primary series. Overall VE was 57\% (95\% CI 42\%, 68\%). VE was $65 \%(95 \%$ CI 54\%, 79\%) in mixed vaccine cohort and $52 \%(95 \%$ CI $30 \%, 68 \%)$ in those who received only acellular vaccine; the difference was not statistically significant. In both the groups, VE waned similarly and significantly $(P<0.01)$ after 2 years; overall VE was $69 \%$ (95\% CI 54\%, 79\%) during first two years post-vaccination and $34 \%(95 \%$ CI $1 \%, 55 \%)$ beyond two years of receiving vaccine.

There is a need to develop new pertussis vaccines and strategies for prolonged immunity against pertussis.

'Sexting' behavior among youth (JAMA Pediatr. 2018;172:327-35)

Current easy access to smartphones has provided adolescents the opportunity to explore their emerging sexuality in novel ways. Sexting is sharing of sexually explicit video, images or messages through electronic means, and may be associated with emotional problems such as cyberbullying and revenge porn, and other high-risk behaviors. This systematic review of 39 international research studies conducted from 2008 to 2016, with 110,380 adolescent participants, estimated the mean prevalence for sending and receiving 'sexts' as $14.8 \%$ (95\% CI 12.8\%, 16.8\%) and 27.4\% (95\% CI 23.1\%, 31.7\%) respectively. The prevalence was equal in both sexes and increased with age, time and with the device being used (more by smartphones compared to computers). The prevalence of forwarding a 'sext' without consent was $12.0 \%$ (95\% CI $8.4 \%, 5.6 \%)$ and of having a 'sext' forwarded without consent was $8.4 \% \quad(95 \%$ CI $4.7 \%, 12.0 \%)$. Pediatricians, parents, teachers, lawyers and policy makers should be aware about the prevalence of 'sexting' and its behavioral ramifications to deal with the issue in a developmentally appropriate manner. In India, currently, 'sexting' is a crime under POCSO Act.

\author{
Online game shutdown policy and its impact on \\ adolescents ( $J$ Adolesc Health. 2018;62:548-55)
}

Gaming disorder is included as an addictive disorder in the recently released draft revision of International Classification of Diseases-11 (ICD-11). Its prevalence is increasing worldwide, especially among adolescents. In 2011, the South Korean government banned online gaming from 12 AM to 6 AM for those aged $<15$ years in order to reduce internet addiction. This study analyzed data of 243,957 adolescents using stratified multistage cluster sampling design from the Korean Youth Risk Behaviour Web based Survey from 201115 to evaluate the effect of this shutdown. It did not show substantial decrease in daily internet usage or internet addiction rates, and did not result in increase in sleeping hours. Female adolescents, those with poor academic performance, and those with low exercise levels showed longer lasting initial declines in internet usage time. This study suggests that a simple shutdown policy fails to deal with the menace of internet addiction in the population, other effective clinical and public health measures should be devised.

Strategies forself-regulation in children and adolescents (JAMA Pediatr. 2018;172:566-75)

Self-regulation is the ability to control emotions and impulses, develop healthy interpersonal relations and to promote self-directed learning. This review, the first of its kind, evaluated 49 randomized clinical trials conducted between 1977 and 2017 with 23,098 participants, aged 2 to 17 years. Consistent improvement in self-regulationwas reported in 76\% curriculum-based interventions, 50\% mindfulness and yoga interventions (especially effective in adolescents), 56\% family-based programs, 67\% exercisebased programs, and $67 \%$ social and personal skills interventions. Associations of interventions withselfregulation task performance scores showed a positive effect with pooled effect size of 0.42 (95\% CI $0.32,0.53)$. Positive associations were also reported on academic achievement, substance abuse, conduct disorders, social skills, depression, behavioral problems, and on reducing school suspension. Pediatricians should partner with parents and take the lead in advocacy for school-based curriculum programs promoting life skills, physical activity, yoga and meditation to enhance biopsychosocial well-being in children and adolescents.

Preeti M Galagali drpgalagali@gmail.com 